\title{
Nível de Atividade Física, Participação e Qualidade de Vida de Brasileiros e Portugueses com Deficiência Física
}

\author{
Fernanda Carolina Toledo da Silva ${ }^{1}$, Lígia Maria Presumido Braccialli ${ }^{2}$, José Pedro Ferreira ${ }^{3}$
}

\begin{abstract}
RESUMO
A aptidão física e o estado de saúde estão relacionados à prática regular de atividade física que proporciona melhores níveis de resistência cardiorrespiratória, força muscular, entre outros benefícios, aos indivíduos fisicamente ativos. A atividade física adaptada pode auxiliar na prevenção de contraturas ou deformidades. O objetivo deste estudo foi verificar o nível de participação, de atividade física e de qualidade de vida de brasileiros e portugueses com deficiência física. Participaram deste estudo 53 brasileiros e 67 portugueses com deficiência física com idade entre 19 e 59 anos, dos gêneros masculino e feminino, com deficiência física, sendo a média de $38,45( \pm 12,39)$ anos para os brasileiros e $38,01( \pm 11,69)$ anos para os portugueses. Foram utilizados três questionários: PASIPD, WHOQOL-abreviado e Escala de Participação. Com relação ao nível de atividade física, os brasileiros atingiram a pontuação média de 23,54 e os portugueses $19,07 \mathrm{MET} \mathrm{hr} / \mathrm{d}$. Quanto ao nível de qualidade de vida, os portugueses obtiveram melhores pontuações que os brasileiros em todos os domínios do questionário. Na escala de participação, a média dos brasileiros foi 16,29 e dos portugueses 19,40 pontos, destacando-se que ambos encontram-se na segunda classe: restrição leve na participação. Com o presente estudo foi identificado que o nível de atividade física e o nível de participação dos brasileiros foram melhores que os dos portugueses, no entanto não foi encontrada diferença estatisticamente significante. Nos domínios da qualidade de vida, os brasileiros pontuaram menos que os portugueses, e houve diferença estatística significante nos domínios ambiental e físico, indicando que os portugueses percebem melhor sua qualidade de vida ambiental e física que os brasileiros.
\end{abstract}

Palavras-chave: Deficiência física. Nível de atividade física. Participação. Qualidade de vida. Atividade física adaptada.

\section{LEVEL OF PHYSICAL ACTIVITY, PARTICIPATION AND QUALITY OF LIFE OF} BRAZILIANS AND PORTUGUESES WITH PHYSICAL DISABILITY

\section{ABSTRACT}

Physical fitness and health status are related to the regular practice of physical activity that provides better levels of cardiorespiratory endurance, muscular strength, among other benefits, to physically active individuals. Adapted physical activity may help prevent contractures or deformities. The aim of this study was to verify the level of participation, physical activity and quality of life of Brazilians and Portuguese with physical disability. Participated in this study were 53 Brazilians and 67 Portuguese with physical disabilities aged between 19 and 59 years. Three questionnaires were used: PASIPD, WHOQOL-Bref and Participation Scale. Regarding the level of physical activity, the Brazilians reached the average score of 23.54 and the Portuguese 19.07 MET hr/d. Regarding the level of quality of life, the Portuguese obtained better scores than the Brazilians in all domains of the questionnaire. In the participation scale, the average of Brazilians was 16.29 and the Portuguese 19.40 points, both of which are in the second class: mild restriction. With this study it was identified that the level of physical activity and the level of participation of the Brazilians were better than those of the Portuguese. However, no statistically significant difference was found. Quality of life domains, Brazilians scored less than the Portuguese, and there was a significant statistical difference in the environmental and physical domains, indicating that the Portuguese perceive better their environmental and physical quality of life than the Brazilians.

Keywords: Physical disability. Physical activity level. Participation. Quality of life. Adapted physical activity. 


\section{INTRODUÇÃO}

A aptidão física e o estado de saúde estão relacionados à prática regular de atividade física que proporciona melhores níveis de resistência cardiorrespiratória, força muscular, entre outros benefícios, aos indivíduos fisicamente ativos (PHYSICAL..., 2008). O Conselho Federal de Educação Física, em sua resolução no 206/2010, artigo 9으, parágrafo 1 , define atividade física como "[...] todo movimento corporal humano, que resulta num gasto energético acima dos níveis de repouso, caracterizado pela atividade do cotidiano e pelos exercícios físicos. Trata-se de comportamento inerente ao ser humano com características biológicas e socioculturais [...]" (CONFEF, 2010, p. 3)

A Organização Mundial da Saúde (WHO, 2018) lançou uma campanha para aumentar a prática de atividade física em todo o mundo, uma vez que $1 \mathrm{em}$ cada 4 adultos não segue as recomendações para a prática de atividade física. Além disso, o nível de atividade física entre pessoas com deficiência geralmente é menor do que em pessoas sem deficiência (SERON; ARRUDA; GREGUOL, 2015).

Strapasson, Martins e Schutz (2002) encontraram em seu estudo que o programa de Educação Física Adaptada melhorou a amplitude de movimento dos participantes com paralisia cerebral. Nesse sentido, fica evidente que a prática de atividade física adaptada pode auxiliar na prevenção de contraturas e deformidades, além de desenvolver propostas de trabalho com caráter lúdico.

Realizar atividade física regularmente proporciona muitos benefícios para todas as populações e pode estar associada ao nível de participação na atividade e influenciar a qualidade de vida dos seus participantes. Noce, Simim e Mello (2009) verificaram o efeito da atividade física na qualidade de vida de pessoas com deficiência física. Foi utilizado o WHOQOL-abreviado com pessoas com deficiência física divididos em dois grupos, ativos e sedentários, e os resultados indicaram que nos quatro domínios do questionário - psicológico, social, ambiental e físico - os participantes ativos obtiveram melhor qualidade de vida do que os sedentários, observando-se que o melhor escore dos participantes do grupo ativo foi no domínio psicológico e do grupo sedentário foi no domínio social.

Na pesquisa de Melo (2014) foi verificada a relação entre o nível de atividade física e a qualidade de vida de amputados de membros inferiores, e foi encontrado que os indivíduos ativos possuíam melhor qualidade de vida nos domínios físico e psicológico que os sedentários. Em um estudo que avaliou a quali- dade de vida de esportistas e não esportistas amputados, foi encontrada diferença significante em todos os domínios do instrumento utilizado, sendo percebida melhor qualidade de vida nos que realizavam a prática esportiva (ZANONA, 2014).

A partir do contexto de que o nível de atividade física de pessoas com deficiência física é menor do que o de pessoas sem deficiência, o que também pode ocorrer quanto ao nível de participação e de qualidade de vida das pessoas com deficiência física, destaca-se a importância da investigação sobre o nível de participação, de atividade física e de qualidade de vida de brasileiros e portugueses com deficiência física. Assim, o objetivo deste estudo foi verificar o nível de participação, de atividade física e de qualidade de vida de brasileiros e portugueses com deficiência física.

\section{MÉTODO}

Este estudo faz parte de uma pesquisa de Doutorado, cujo projeto foi aprovado pelo Comitê de Ética em Pesquisa sob o parecer número $1.503 .491 \mathrm{em}$ abril de 2016, com adendo número 2.394.956 em novembro de 2017 e CAAE 53821715.9.0000.5406. Os participantes assinaram um Termo de Consentimento Livre e Esclarecido.

Trata-se de uma pesquisa quantitativa, a qual apresenta dados que podem ser quantificados, coletados com auxílio de instrumentos padronizados, trazendo a descrição de causas de um fenômeno por meio de linguagem matemática (FONSECA, 2002). Além disso, a pesquisa quantitativa utiliza procedimentos estruturados e instrumentos formais para coleta de dados e analisa os dados numéricos mediante procedimentos estatísticos (GERHARDT; SILVEIRA, 2009).

Para seleção dos participantes foi realizado contato com instituições que atendem pessoas com deficiência física, e posteriormente foi feito contato com os indivíduos com deficiência física. Foram participantes da pesquisa aqueles que demonstraram interesse em fazer parte do estudo. Assim, participaram 53 brasileiros e 67 portugueses com idade entre 19 e 59 anos, do gênero masculino e feminino, com deficiência física, sendo a média de $38,45( \pm 12,39)$ anos para os brasileiros e $38,01( \pm 11,69)$ anos para os portugueses. Eles foram divididos em três grupos conforme a faixa etária, de acordo com a escala de classificação da Organização Mundial da Saúde: os participantes de 18 a 30 anos foram considerados para a faixa etária ida- 
de adulta jovem, os de 31 a 45 anos foram incluídos na idade madura e dos 46 aos 59 anos para idade de mudança.

A coleta de dados ocorreu em diferentes locais no Brasil e em Portugal. No Brasil deu-se no Centro de Estudos em Educação e Saúde (Cees) da Universidade Estadual Paulista (Unesp-Marília), na Clínica-Escola do Curso de Fisioterapia da Universidade de Marília (Unimar) e na Associação Mariliense de Esportes Inclusivos (Amei), localizados na cidade de Marília/SP; na Praça Paradesportiva de Bauru e no Departamento de Educação Física da Universidade Estadual Paulista (Unesp-Bauru), na cidade de Bauru/SP. O período de coleta no Brasil foi de outubro 2016 a março de 2017 e de setembro de 2017 a março de 2018.

Em Portugal os dados foram coletados na Associação de Paralisia Cerebral de Coimbra (APCC), na Faculdade de Ciências do Desporto e Educação Física (FCDEF) da Universidade de Coimbra (UC) na cidade de Coimbra, na Universidade do Porto (UP), na cidade do Porto, e em competições de bocha adaptada nas cidades de Barcelos, Santarém e Paredes. O período de coleta em Portugal compreendeu os meses de março a junho de 2017.

Foram utilizados três instrumentos padronizados 1) Escala de Atividade Física para Pessoas com Deficiência Física (PASIPD); 2) World Health Organization Quality of Life (WHOQOL-abreviado); 3) Escala de Participação (Participation Scale - PS). Também foi utilizada uma ficha para preenchimento dos dados pessoais dos participantes.

O instrumento para mensuração do nível de atividade física Physical Activity Scale for Persons with Physical Disabilities - PASIPD (WASHBURN et al., 2002), foi traduzido e adaptado culturalmente para a língua portuguesa do Brasil e traduzido e validado para a língua portuguesa de Portugal.

O instrumento é de autoaplicação e avalia o nível atual de atividade e de exercícios físicos de pessoas com deficiência física. A escala é composta por 13 itens que englobam três temas: 6 questões sobre atividades de lazer, 6 sobre atividades domésticas e 1 questão sobre atividade relacionada ao trabalho. A primeira questão não é pontuada e é usada para habituar o participante ao instrumento. Todas as questões possuem duas partes: a primeira parte relacionada à frequência com que a atividade foi realizada e a segunda relativa ao número de horas despendidas na atividade. Nas questões de 3 a 6 há um espaço direcionado para que a pessoa descreva qual foi o tipo de atividade realizada.
O instrumento para mensuração da qualidade de vida World Health Organization Quality of Life WHOQOL-abreviado (THE WHOQOL GROUP, 1994) foi traduzido e validado para a língua portuguesa do Brasil (FLECK, 2000) e traduzido e validado para a língua portuguesa de Portugal (CANAVARRO et al., 2006). Trata-se de uma versão abreviada do WHOQOL-100, de autoaplicação, composto pelas 26 perguntas que obtiveram os melhores desempenhos psicométricos distribuídas em quatro domínios: físico, psicológico, relações sociais e ambiente (FLECK, 2000).

No domínio psicológico (Psych) são incluídos questionamentos sobre os sentimentos positivos, pensar, aprender, memória, concentração, autoestima, imagem corporal, aparência, sentimentos negativos, espiritualidade, religião e crenças pessoais. 0 domínio relações sociais (Social) abrange questões sobre as relações pessoais, suporte social e atividade sexual. No domínio ambiente (Envir) são abordadas questões relacionadas à segurança e proteção, ambiente no lar, recursos financeiros, cuidados de saúde, oportunidade de adquirir novas informações e habilidades, participação em atividades de lazer, trânsito e transporte. O domínio físico (Phys) inclui questões sobre dor e desconforto, energia e fadiga, sono e repouso, mobilidade, atividades de vida diária, dependência de medicação ou tratamentos e capacidade para o trabalho. As 26 questões são fechadas e possuem cinco possibilidades de escolha de respostas em escala do tipo Likert (FLECK, 2000).

Em relação ao instrumento para mensuração da participação, Participation Scale - PS (VAN BRAKEL et al., 2006), o estudo de desenvolvimento da escala foi realizado simultaneamente em seis idiomas, em três países: Nepal, Índia e Brasil. A escala foi adequada culturalmente para a população portuguesa para realização deste estudo. $O$ instrumento foi construído a partir de seis termos de referência, tais como: a) ser baseado nos domínios de participação da CIF; b) ser de natureza transcultural; c) ser de percepção do respondente; d) ser genérico quanto à sua natureza; e) ser adequado para entrevistadores inexperientes e f) usar o conceito de comparação entre pares. A escala possui 18 questões, é um instrumento baseado em entrevista e possibilita verificar a percepção do respondente com condição adversa de saúde ou com deficiência quanto às suas restrições na participação em relação a seus pares. Antes de iniciar a reposta dos itens da escala o participante deve se comparar a uma pessoa com semelhanças em todos os aspectos, exceto pela doença ou deficiência. Quando o participante responde "sim" à questão ela não é pontuada; quan- 
do é respondido "às vezes", "não" ou "irrelevante, eu não quero, eu não preciso", deve ser apontado em que grau isso representa um problema em sua vida, pontuando 1 quando "não é problema", 2 quando o problema é "pequeno", 3 quando o problema é "médio" e 5 quando o problema é considerado "grande".

Para realização da coleta dos dados foram feitos contatos com as instituições que atendem pessoas com deficiência física, no Brasil e em Portugal, para então realizar a comunicação com os participantes.

As coletas ocorreram por meio de entrevista ou eletronicamente, ressaltando-se que quanto aos participantes com os quais não foi possível realizar a entrevista, foi feito o primeiro contato pessoalmente e em seguida enviado o acesso eletrônico dos questionários. Já os participantes com os quais não foi realizado contato pessoalmente, foi feita a explicação da pesquisa virtualmente, expondo os objetivos e a maneira como ocorreria a participação na pesquisa.

Assim, os questionários impressos foram preenchidos pelo próprio participante, ou pela pesquisadora, quando respondidos por meio de entrevista entre ambos pessoalmente. E quando não foi possível realizar o preenchimento dos questionários pessoalmente com o participante, foram utilizados os questionários eletrônicos na versão brasileira e na versão portuguesa, por meio do formulário on-line Google forms. 0 link de acesso aos instrumentos disponíveis eletronicamente foi enviado por e-mail ou pelas redes sociais (Whatsapp ou Facebook) do participante. Os participantes nesta condição preencheram os formulários on-line e a pesquisadora os recebeu diretamente em seu e-mail.

Para a análise de dados os escores de cada instrumento para cada participante foram inseridos em planilha no software IBM SPSS Statistics.

Para obtenção do escore da escala do nível de atividade física Pasipd há itens multiplicadores para cada questão de 2 a 13 e uma tabela com valores a serem pontuados conforme a frequência de dias da semana e do número de horas de realização da atividade física da questão. Após a multiplicação de cada questão, é realizada a soma delas e obtém-se o escore total em Equivalente Metabólico - MET hr/d, ou seja, o gasto energético diário em atividades físicas, sendo o valor máximo do instrumento de 199,5 MET hr/d. Quanto maior a pontuação obtida no escore total, maior o nível de atividade física do participante com deficiência física.
O escore do questionário de qualidade de vida WHOQOL-abreviado é obtido pela utilização de uma sintaxe para o cálculo dos domínios diretamente no software SPSS. O escore do instrumento é obtido separadamente em cada domínio: psicológico, social, ambiental e físico, e pode atingir valores de 0 a 100 . Quanto maior a pontuação, melhor qualidade de vida da pessoa com deficiência física para cada domínio.

O escore da escala de participação ? PS - é obtido pela soma dos 18 itens, e conforme a pontuação atingida é classificado o nível de participação do respondente. A classificação inicia-se em nenhuma restrição relevante quando o participante atinge até 12 pontos; restrição leve quando atinge de 13 a 22 pontos; restrição moderada quando pontua de 23 a 32; restrição severa quando a pontuação atingida é de 33 a 52 e restrição extrema quando atinge 53 pontos ou mais.

Os dados referentes à coleta realizada nos dois países foram tabulados em planilhas específicas e comparados entre si e entre países. Os dados foram agrupados em tabelas, por meio de frequências, absolutas e percentuais, número de indivíduos (n), média e desvio-padrão (DP).

Foi verificada a normalidade dos dados, no entanto nem todas as variáveis atingiram significância nos testes Kolmogorov-Smirnov e Shapiro-Wilk, o que levou a considerar a amostra não paramétrica. Para as variáveis numéricas verificou-se a similaridade entre os grupos por meio dos testes $U$ de Mann-Whitney e Kruskal-Wallis. Adotou-se, para todos os testes, o nível de significância de $5 \%$ de probabilidade para a rejeição da hipótese de nulidade.

\section{RESULTADOS E DISCUSSÃO}

A idade média dos participantes brasileiros foi de $38,45( \pm 12,39)$ anos e dos portugueses de 38,01 $( \pm 11,69)$ anos. Na Tabela 1 verifica-se a distribuição dos dados quanto ao gênero, à faixa etária e ao diagnóstico dos participantes.

Tabela 1 - Frequência absoluta e relativa do gênero, faixa etária e diagnóstico dos participantes

\begin{tabular}{c|c|c|c}
\hline Gênero & \multicolumn{1}{c}{ BR n (\%) } & \multicolumn{1}{c}{ PT n (\%) } & \multicolumn{1}{c}{ TOTAL n (\%) } \\
Masculino & $34(64,2)$ & $43(64,2)$ & $77(64,2)$ \\
Feminino & $19(35,8)$ & $24(35,8)$ & $43(35,8)$ \\
Total & $53(100)$ & $67(100)$ & $120(100)$ \\
\hline Faixa etária & BR n (\%) & PT n (\%) & TOTAL n (\%) \\
Adulta jovem & $17(32,1)$ & $24(35,8)$ & $41(34,2)$ \\
Idade madura & $17(32,1)$ & $23(34,3)$ & $40(33,3)$ \\
Idade de mudança & $19(35,8)$ & $20(29,9)$ & $39(32,5)$ \\
Total & $53(100)$ & $67(100)$ & $120(100)$ \\
\hline
\end{tabular}




\begin{tabular}{c|c|c|c}
\hline Diagnóstico & BR n (\%) & PT n (\%) & TOTAL $n(\%)$ \\
Paralisia Cerebral & $4(7,6)$ & $28(41,8)$ & $32(26,7)$ \\
Amputação, lesão & $18(33,9)$ & $20(29,9)$ & $38(31,7)$ \\
medular e similares & & & \\
Outros & $31(58,5)$ & $19(28,4)$ & $50(41,7)$ \\
Total & $53(100)$ & $67(100)$ & $120(100)$ \\
\hline
\end{tabular}

Fonte: Elaborado pelos autores. Legenda: BR - participantes brasileiros; PT - participantes portugueses.

Na Tabela 2 verificou-se que a média do nível de participação dos brasileiros foi de 16,29 pontos e portugueses 19,40 pontos. Embora a pontuação dos brasileiros seja menor que a dos portugueses, os dois grupos apresentam classificação entre 13 e 22 pontos, o que indica que ambos possuem restrição leve na participação. Os brasileiros apresentaram maior nível participação e de atividade física (23,54 MET hr/d) que os portugueses (19,07 MET hr/d), e apresentaram menor qualidade de vida nos quatro domínios (psicológico, social, ambiental e físico), no entanto foi verificada diferença estatística significante entre a amostra brasileira e a portuguesa apenas para os domínios ambiental e físico da qualidade de vida, tendo os portugueses melhor qualidade de vida nos domínios ambiental e físico do que os brasileiros (Tabela 2).

Tabela 2 - Teste U de Mann-Whitney de amostras independentes

\begin{tabular}{cccc}
\hline & $\begin{array}{c}\text { BR - Média } \\
\pm \text { desvio padrão }\end{array}$ & $\begin{array}{c}\text { PT - Média } \pm \text { desvio } \\
\text { padrão }\end{array}$ & $\mathrm{P}$ \\
PS & $16,29 \pm 12,26$ & $19,40 \pm 15,98$ & 0,411 \\
PASIPD & $23,54 \pm 20,32$ & $19,07 \pm 19,84$ & 0,098 \\
PSYCH & $68,64 \pm 12,55$ & $72,32 \pm 17,34$ & 0,067 \\
SOCIAL & $65,91 \pm 15,65$ & $68,66 \pm 19,57$ & 0,389 \\
ENVIR & $59,54 \pm 13,91$ & $66,79 \pm 15,53$ & $0,014^{*}$ \\
PHYS & $59,35 \pm 17,04$ & $67,70 \pm 15,09$ & $0,008^{*}$ \\
\hline
\end{tabular}

Fonte: Elaborado pelos autores. *significância $p \leq 0,05$.

Legenda: BR: participantes brasileiros; PT: participantes portugueses; PS: Escala de Participação; PASIPD: Escala do nível de atividade física de pessoas com deficiência física; PSYCH: domínio psicológico do WHOQOL-abreviado; SOCIAL: domínio social do WHOQOL-abreviado; ENVIR: domínio ambiental do WHOQOL-abreviado; PHYS: domínio físico do WHOQOL-abreviado. Escore PS: nenhuma restrição relevante: até 12 pontos; restrição leve: 13 a 22 pontos; restrição moderada: 23 a 32 pontos; restrição severa: 33 a 52; restrição extrema: 53 pontos ou mais.

Com relação ao nível de atividade física das pessoas com deficiência física, Buffart et al. (2008a) encontraram que $39 \%$ dos seus participantes com mielomeningocele são inativos e $37 \%$ extremamente inativos, além de apresentarem aptidão física baixa e obesidade. Além disso, foi encontrado que os participantes com mielomeningocele com maior nível de deambulação são mais ativos fisicamente durante o dia, têm maior nível de aptidão física e menos gordura corporal comparados às pessoas com menor nível de deambulação.
Outro estudo desenvolvido por Buffart et al. (2008b) também encontrou que a maioria dos adolescentes e jovens adultos com mielomeningocele apresentou baixa aptidão física relacionada à saúde. Comparado à população geral e a outros grupos de pacientes, eles têm baixa capacidade aeróbica (BUFFART et al., 2008b).

Quanto à qualidade de vida, escoceses amputados de membros inferiores tiveram escores piores quanto ao nível de qualidade de vida do que brasileiros e portugueses nos domínios psicológico $(64,72)$, social $(62,24)$ e físico $(56,32)$, mas melhores em relação ao domínio ambiental $(72,20)$ (DEANS; McFADYEN; ROWE, 2008). No estudo de Lima, Ribeiro e Tonello (2012) foram identificados melhores níveis de qualidade de vida de brasileiros com deficiência física da região de Minas Gerais nos domínios psicológico $(75,78)$ e social $(75,08)$ comparados aos brasileiros e portugueses do presente estudo. Os autores identificaram diferença significante entre participantes e não participantes de atividades de lazer com deficiência física nos quatro domínios do questionário de qualidade de vida, ressaltando-se que os participantes percebem melhor sua qualidade de vida do que os não participantes (LIMA; RIBEIRO; TONELLO, 2012).

Quanto à diferença estatística significante encontrada entre brasileiros e portugueses nos domínios ambiental e físico, ressalta-se a pesquisa de Noce, Simim e Mello (2009), na qual encontraram escores da qualidade de vida de ativos com deficiência física maiores do que dos sedentários com deficiência física, sendo significativamente maiores nos domínios físico e psicológico para os ativos.

No teste $U$ de Mann-Whitney foi verificada diferença significante entre gêneros no domínio psicológico da qualidade de vida da amostra portuguesa. Não houve diferença significante estatisticamente entre o gênero masculino e feminino da amostra brasileira, mas os dados apontaram que os homens brasileiros apresentaram piores resultados com relação ao nível de participação, uma vez que quanto maior a pontuação na escala de participação, maiores as restrições na participação, semelhante escore quanto ao nível de atividade física das mulheres, e melhores escores do que as mulheres nos domínios psicológico e físico de qualidade de vida (Tabela 3).

Os homens portugueses também apresentaram piores pontuações no nível de participação do que as mulheres, mas obtiveram melhores pontuações do que as mulheres portuguesas no nível de atividade física. Em todos os domínios de qualidade de vida os portugueses atingiram pontuações menores que as portuguesas, mas houve diferença significativa entre 
Tabela 3 - Teste U de Mann-Whitney de amostras independentes para gênero

\begin{tabular}{lcccccc}
\hline & $\begin{array}{c}\text { Masculino - BR } \\
\text { Média } \pm \text { desvio } \\
\text { padrão }\end{array}$ & $\begin{array}{c}\text { Feminino - BR } \\
\text { Média } \pm \text { desvio } \\
\text { padrão }\end{array}$ & $p-B R$ & $\begin{array}{c}\text { Masculino - PT } \\
\text { Média } \pm \text { desvio } \\
\text { padrão }\end{array}$ & $\begin{array}{c}\text { Feminino - PT } \\
\text { Média } \pm \text { desvio } \\
\text { padrão }\end{array}$ & $\begin{array}{c}p-\text { PT } \\
16,83 \pm 11,01\end{array}$ \\
PS & $17,35 \pm 12,46$ & $15,21 \pm 12,40$ & 0,571 & $20,84 \pm 18,14$ & 0,661 & 0,556 \\
PASIPD & $23,59 \pm 21,50$ & $23,44 \pm 18,57$ & 0,643 & $21,41 \pm 22,89$ & $14,87 \pm 12,02$ & $0,011 *$ \\
PSYCH & $68,63 \pm 14,77$ & $67,76 \pm 7,71$ & 0,215 & $71,03 \pm 16,81$ & $74,65 \pm 18,39$ & 0,685 \\
SOCIAL & $64,46 \pm 18,38$ & $69,30 \pm 7,88$ & 0,298 & $64,54 \pm 21,14$ & $76,04 \pm 13,97$ & 0,458 \\
ENVIR & $58,82 \pm 14,89$ & $59,54 \pm 12,52$ & 0,915 & $65,99 \pm 17,09$ & $68,23 \pm 12,45$ & 0,328 \\
PHYS & $61,03 \pm 17,19$ & $54,51 \pm 16,01$ & 0,096 & $66,78 \pm 16,74$ & $69,35 \pm 11,72$ & 0.72 \\
\hline
\end{tabular}

Fonte: Elaborado pelos autores. *significância $\mathrm{p} \leq 0,05$.

Legenda: BR: participantes brasileiros; PT: participantes portugueses; PS: Escala de Participação; PASIPD: Escala do nível de atividade física de pessoas com deficiência física; PSYCH: domínio psicológico do WHOQOL-abreviado; SOCIAL: domínio social do WHOQOL-abreviado; ENVIR: domínio ambiental do WHOQOL-abreviado; PHYS: domínio físico do WHOQOL-abreviado. Escore PS: nenhuma restrição relevante: até 12 pontos; restrição leve: 13 a 22 pontos; restrição moderada: 23 a 32 pontos; restrição severa: 33 a 52; restrição extrema: 53 pontos ou mais.

gêneros apenas no domínio psicológico da amostra portuguesa. Assim sendo, as mulheres percebem meIhor sua qualidade de vida psicológica do que os homens portugueses (Tabela 3).

Assim como a amostra portuguesa, embora não tenha sido realizado teste estatístico, Silva et al. (2011) também encontraram melhores níveis de qualidade de vida entre mulheres no domínio psicológico. Os homens da pesquisa de Silva et al. (2011) percebem melhor sua qualidade de vida social e ambiental, ao contrário dos escores encontrados na amostra brasileira e portuguesa deste estudo.

No teste de Kruskal-Wallis foi encontrada diferença significativa entre as idades no domínio físico da qualidade de vida da amostra brasileira e no domínio psicológico da qualidade de vida da amostra portuguesa (Tabela 4).
Os dados brasileiros apontaram que os participantes classificados como idade de mudança apresentaram maiores restrições na participação e menores níveis de atividade física. Nos domínios da qualidade de vida dos brasileiros foi encontrada, para o domínio psicológico, a mesma percepção entre os participantes classificados em idade adulta jovem e idade madura, destacando-se que estes dois grupos percebem melhor sua qualidade de vida psicológica que o grupo de idade de mudança. No domínio social a faixa etária que obteve melhor percepção em sua qualidade de vida foi o grupo mais velho, de idade de mudança. No domínio ambiental a melhor percepção foi do grupo idade madura. E no domínio físico os participantes classificados como idade de mudança tiveram pior

Tabela 4 - Teste Kruskal-Wallis de amostras independentes para idade

\begin{tabular}{|c|c|c|c|c|c|c|c|c|}
\hline & $\begin{array}{c}\text { Idade adulta } \\
\text { jovem - BR } \\
\text { Média } \pm \text { desvio } \\
\text { padrão }\end{array}$ & $\begin{array}{c}\text { Idade madura } \\
\text { - BR } \\
\text { Média } \pm \text { desvio } \\
\text { padrão }\end{array}$ & $\begin{array}{c}\text { Idade de } \\
\text { mudança - BR } \\
\text { Média } \pm \text { desvio } \\
\text { padrão }\end{array}$ & $p-B R$ & $\begin{array}{c}\text { Idade adulta } \\
\text { jovem - PT } \\
\text { Média } \\
\text { 土desvio } \\
\text { padrão }\end{array}$ & $\begin{array}{c}\text { Idade } \\
\text { madura - PT } \\
\text { Média } \\
\pm \text { desvio } \\
\text { padrão }\end{array}$ & $\begin{array}{c}\text { Idade de } \\
\text { mudança - PT } \\
\text { Média } \\
\text { 土desvio } \\
\text { padrão }\end{array}$ & $p-P T$ \\
\hline$P S$ & 7 & 20 & 3 & 0, & $15,79 \pm 10,88$ & $22,61 \pm 20,54$ & $20,05 \pm 15,04$ & 0,427 \\
\hline PASIPD & $32,15 \pm 24,07$ & $23,38 \pm 21,39$ & $15,98 \pm$ & 0,077 & $14,56 \pm 14,62$ & $24,85 \pm 20,56$ & $17,83 \pm 23,50$ & 0,087 \\
\hline PSYCH & $68,63 \pm 10,53$ & $68,63 \pm 14,89$ & $67,76 \pm 12,79$ & 0,675 & $74,48 \pm 18,52$ & $73,73 \pm 17,47$ & $68,13 \pm 15,78$ & $0,006^{*}$ \\
\hline SOCIAL & $66,18 \pm 18,03$ & $65,69 \pm 17,40$ & $66,67 \pm 11,78$ & 0,881 & $75,35 \pm 14,43$ & $71,02 \pm 21,74$ & $57,92 \pm 18,63$ & 0,065 \\
\hline ENVIR & $56,98 \pm 16,29$ & $62,13 \pm 14,23$ & $58,22 \pm 11,56$ & 0,457 & $71,62 \pm 15,66$ & $66,98 \pm 16,15$ & $60,78 \pm 13,08$ & 0,327 \\
\hline PHYS & $65,13 \pm 14,26$ & $58,40 \pm 19,92$ & $53,19 \pm 14,91$ & 0,051 & $69,73 \pm 15,58$ & $68,79 \pm 15,31$ & $63,93 \pm 14,28$ & 0,288 \\
\hline
\end{tabular}

Fonte: Elaborado pelos autores. *significância $\mathrm{p} \leq 0,05$.

Legenda: BR: participantes brasileiros; PT: participantes portugueses; PS: Escala de Participação; PASIPD: Escala do nível de atividade física de pessoas com deficiência física; PSYCH: domínio psicológico do WHOQOL-abreviado; SOCIAL: domínio social do WHOQOL-abreviado; ENVIR: domínio ambiental do WHOQOL-abreviado; PHYS: domínio físico do WHOQOL-abreviado. Escore PS: nenhuma restrição relevante: até 12 pontos; restrição leve: 13 a 22 pontos; restrição moderada: 23 a 32 pontos; restrição severa: 33 a 52; restrição extrema: 53 pontos ou mais. 
percepção da qualidade de vida, revelando-se este o domínio que apresentou diferença estatística significativa na amostra brasileira (Tabela 4).

Com os dados portugueses foi verificado que os participantes classificados em idade de mudança tiveram mais restrições na participação. O nível de atividade física dos portugueses é maior na faixa etária entre 31 e 45 anos, o grupo de idade madura. E em todos os domínios da qualidade de vida os participantes mais velhos tiveram pior escore, embora o domínio que apresentou diferença estatisticamente significante foi o psicológico (Tabela 4).

\section{CONCLUSÕES}

Com o presente estudo foi identificado que os níveis de participação e de atividade física dos brasileiros foram melhores que dos portugueses, no entanto não foi encontrada diferença estatisticamente significante. Em relação à qualidade de vida os brasileiros pontuaram menos que os portugueses, e houve diferença estatística significante para os domínios ambiental e físico, indicando que os portugueses percebem melhor sua qualidade de vida ambiental e física que os brasileiros.

Com relação às análises por nacionalidade dos participantes, foi encontrado que a amostra brasileira apresentou diferença significante para o domínio físico de qualidade de vida relacionado à idade dos participantes. A amostra portuguesa apresentou diferença significante para o domínio psicológico da qualidade de vida com relação ao gênero e à idade.

\section{REFERÊNCIAS}

BUFFART, L. et al. Triad of Physical Activity, Aerobic Fitness and Obesity in Adolescents and Young Adults with Myelomeningocele. In: BUFFART, L. Physical activity and fitness in adolescents and young adults with myelomeningocele. 2008. 191f. Dissertação (Mestrado) - Erasmus University Rotterdam, Rotterdam, 2008a. Cap. 2.

BUFFART, L. et al. Health-Related Physical Fitness of Adolescents and Young Adults with Myelomeningocele. In: BUFFART, L. Physical activity and fitness in adolescents and young adults with myelomeningocele. 2008. 191f. Dissertação (Mestrado) - Erasmus University Rotterdam, Rotterdam, 2008b. Cap. 3.

CANAVARRO, M. C. et al. Desenvolvimento do instrumento de avaliação da qualidade de vida da Organização Mundial de Saúde (WHOQOL-100) para Português de Portugal. Psiquiatria Clínica, n. 27, v. 1, p. 15-23, 2006.

CONFEF. Resolução no 206 de 7 de novembro de 2010. Aprova o Estatuto do Conselho Federal de Educação Física - Confef. Rio de Janeiro, RJ, 2010.
DEANS, S. A.; MCFADYEN, A. K.; ROWE, P. J. Physical activity and quality of life: a study of a lower-limb amputee population. Prosthetics and Orthotics International, v. 32, n. 2, p. 186-200, jun. 2008.

FLECK, M. P. A. et al. Aplicação da versão em português do instrumento abreviado de avaliação da qualidade de vida "WHOQOL-bref". Revista de Saúde Pública. v. 34, n. 2, p. 178-183, abr. 2000.

FONSECA, J. J. S. Metodologia da pesquisa científica. Fortaleza: UEC, 2002.

GERHARDT, T. E.; SILVEIRA, D. T. Métodos de pesquisa. Universidade Aberta do Brasil - UAB/UFRGS. Porto Alegre: Editora da UFRGS, 2009.

LIMA, R. M.; RIBEIRO, P. R. Q.; TONELLO, M. G. M. Percepção da qualidade de vida por pessoas com deficiências físicas praticantes e não praticantes de atividades de lazer. Licere, Belo Horizonte, v. 15, n. 4, dez. 2012.

MELO, V. H. Fatores relacionados com nível de atividade física em indivíduos amputados e a relação entre nível de atividade física com a qualidade de vida. 2014. 64f. Dissertação (Mestrado em Educação Física) - Universidade Federal de Sergipe, São Cristóvão, 2014.

NOCE, F.; SIMIM, M. A. M.; MELLO, M. T. A percepção de qualidade de vida de pessoas portadoras de deficiência física pode ser influenciada pela prática de atividade física? Rev. Bras. Med. Esporte, v. 15, n. 3, maio/jun. 2009.

PHYSICAL ACTIVITY GUIDELINES FOR AMERICANS. Be active, healthy and happy! U.S. Department of Health and Human Services. Washington, D. C. 2008.

SERON, B. B.; ARRUDA, G. A.; GREGUOL, M. Facilitadores e barreiras percebidas para a prática de atividade física por pessoas com deficiência motora. Revista Brasileira de Ciências do Esporte, v. 37, n. 3, p. 214-221, 2015.

STRAPASSON, A. M.; MARTINS, S. M. F. C.; SCHUTZ, R. A meIhora da amplitude articular e/ou manutenção dos movimentos em pessoas portadoras de paralisia cerebral espástica através da educação física adaptada. Revista do Centro de Educação, Cadernos, edição n. 19, 2002.

SILVA, R. et al. Physical activity and quality of life of amputees in southern Brazil. Prosthetics and Orthotics International, v. 35, n. 4, p. 432-438, 2011.

THE WHOQOL GROUP. The development of the World Health Organization quality of life assessment instrument (the WHOQOL). In: ORLEY, J.; KUYKEN, W. (eds.). Quality of life assessment: international perspectives. Heidelberg: Springer Verlag, 1994. p. 41-60.

VAN BRAKEL, W. H.; et al. The participation scale: measuring a key concept in public health. Disability and Rehabilitation, v. 28, n. 4, p. 193-203, fev. 2006.

WASHBURN, R. A. et al. The physical activity scale for individuals with physical disabilities: development and evaluation. Arch. Phys. Med. Rehabil., v. 83, Fev. 2002.

WHO. World Health Organization. Global action plan on physical activity 2018-2030: more active people for healthier world. Geneva: World Health Organization, 2018.

ZANONA, A. F. Qualidade de vida e funcionalidade de individuos amputados praticantes e não praticantes de esportes. 2014. 125f. Dissertação (Mestrado em Educação Física) Universidade Federal de Sergipe, São Cristóvão, 2014. 